ROGÉRIO APARECIDO DOS SANTOS

\title{
DESENVOLVIMENTO DE MICROEMULSÕES E SUA TRANSFORMAÇÃO IN SITU EM GÉIS DE FASE LÍQUIDO-CRISTALINA COMO PLATAFORMA PARA LIBERAÇÃO SUSTENTADA DE FÁRMACOS E SEU USO NO TRATAMENTO DO ALCOOLISMO
}

Dissertação apresentada ao Programa de PósGraduação em Farmacologia do Instituto de Ciências Biomédicas da Universidade de São Paulo para obtenção do Título de Mestre em Ciências

Área de Concentração (Farmacologia)

Orientadora: Profa. Dra.Luciana B. Lopes

Co-orientadora: Profa. Dra. Rosana Camarini

Versão parcial 


\section{RESUMO}

Santos, RA.Desenvolvimento de microemulsões e sua transformação in situ em géis de fase líquido-cristalina como plataforma para liberação sustentada de fármacos e seu uso no tratamento do alcoolismo. [Dissertação (mestrado em Farmacologia)]. São Paulo: Instituto de Ciências Biomédicas, Universidade de São Paulo;2017.

Esforços significativos tem sido feitos para desenvolver formulações capazes de sustentar a liberação de fármacos usados no tratamento de doenças crônicas e com baixa adesão, como alcoolismo. Ainda assim, são necessárias formulações simples, seguras, passíveis de auto-administração, que aumentem a taxa de adesão ao tratamento e diminuam o custo da saúde pública. Este estudo focaliza o desenvolvimento de microemulsões que após captação de água in vivo do tecido subcutâneo transformar-se-ão em géis de fase hexagonal para liberação sustentada da naltrexona. Diagramas de fase mostrando a relação, o tipo e proporção de componentes e sistemas formados foram construídos, e três microemulsões à base de (i) monooleína e tricaprilina (ME-MO), (ii) vitamina E TPGS e propilenoglicol (ME-TPGS) e (iii) TPGS e Span (ME-Span) foram selecionadas. Todas apresentaram diâmetro inferior a $150 \mathrm{~nm}$ e comportamento Newtoniano. ME-MO e METPGS originaram fase hexagonal após intumescimento, enquanto que ME-Span originou fase lamelar. Esta última permitiu a incorporação de 3.8-vezes menos naltrexona, e resultou na liberação mais rápida do fármaco $(65 \%$ em 96 h), provavelmente em decorrência da sua menor viscosidade. Baseado na capacidade do gel formado de resistir à diluição, ME-MO foi selecionada para estudos in vivo. Após administração subcutânea, foi observado formação da fase hexagonal em $48 \mathrm{~h}$ e sua persistência por mais de 30 dias em camundongos, promovendo liberação prolongada de Alexa flúor 647 durante esse período. A eficácia da formulação foi avaliada utilizando modelo de preferência condicionada por lugar. Os animais foram distribuídos em quatro grupos, sendo administrado por via subcutânea: 1) solução salina (controle); 2) solução de naltrexona (1 mg/kg) diária por 8 dias (30 min antes da administração de etanol); e 3) ME-MO com $5 \%$ ou $10 \%$ de naltrexona (administração única). Etanol (2 g/kg) ou salina foram administrados por via intraperitoneal antes do experimento. Todos os animais tratados com naltrexona apresentaram menor preferência condicionada ao etanol, sugerindo redução dos efeitos recompensadores da droga. Não observou-se diferença estatisticamente significante entre a solução de naltrexona e ME-MO 5\%, mas o grupo ME-MO 10\% diferiu estatisticamente da solução naltrexona diária e ME-MO 5\%. Os resultados sugerem que o ME-MO 10\% antagonizou a preferência condicionada por lugar induzida pelo etanol. Esses resultados demonstram o potencial de ME-MO como uma plataforma para liberação prolongada de fármacos visando o tratamento de dependência química.

Palavras-chave: Nanocarreador. Liberação sustentada. Alcoolismo. Naltrexona. Preferência Condicionada por Lugar. 


\begin{abstract}
Santos, RA. Development of microemulsions and their in situ transformation in liquidcrystalline phase gels as a platform for sustained release of drugs and their use in the treatment of alcoholism. [Master thesis (Pharmacology)]. São Paulo: Instituto de Ciências Biomédicas, Universidade de São Paulo;2017.
\end{abstract}

Significant efforts have been made to develop formulations capable of sustaining the release of drugs used in the treatment of chronic diseases, but simple formulations that can be easily and self-administered, and cause minimal local reactions are still needed. This study focuses on the development of microemulsions that can swell and transition into nanostructured hexagonal phase gels upon water uptake in vivo as a plataform for sustained release of naltrexone. Microemulsions were prepared using monoolein, vitamin E TPGS (TPGS), BRIJ or Span as structure-forming surfactants in combination with mono or tricaprylin as oil phase, water and propylene glycol as viscosity modifier. Microemulsions composed of (i) monoolein and tricaprylin (ME-MO), (ii) TPGS and propylene glycol (METPGS) and (iii) TPGS and Span (ME-Span) were selected. All microemulsions displayed nanometric size below $150 \mathrm{~nm}$ and rheological behavior consistent with Newtonian systems. Uptake of $20 \%$ or more of water by ME-TPGS and ME-MO led to formation of hexagonal phase while ME-Span resulted in lamellar phase. The later incorporated $3.8 \%$ less naltrexone, and promoted the fastest drug release (approximately 65\% within $96 \mathrm{~h}$ ), most likely due its lower viscosity. ME-MO was selected for further evaluation based on its ability to form gels that resisted dilution. Gels formed in vivo approximately $48 \mathrm{~h}$ after ME-MO subcutaneous administration, and persisted locally for over 30 days providing slow release of Alexa fluor compared to a propylene glycol solution. Formulation efficacy was evaluated using a conditioned place preference model (CPP). Mice were distributed into groups, and subjected to subcutaneous administration of saline (control), $1 \mathrm{mg} / \mathrm{kg}$ naltrexone solution daily (8 days), ME-MO with 5 or $10 \%$ naltrexone (single administration), Alcohol $(2 \mathrm{~g} / \mathrm{kg}$ ) or saline was administered intraperitoneally before CPP training. Statistically significant $(p \leq$ 0.05 ) between groups naltrexone and ME MO solution 5\%. However no statistically significant difference was observed between the solution of naltrexone and ME-MO 5\%. These results support the use of ME-MO as a plataform for sustained release of drugs useful in the treatment of drug addiction.

Keywords: Nanocarrier. Sustained Release. Naltrexone. Alcoholism. Conditioned Place Preference. 


\section{INTRODUÇÃO}

"Beber inicia num ato de

liberdade, caminha para o hábito e, finalmente, afunda na necessidade”.

Benjamin Rush

O álcool é uma substância que acompanha a humanidade desde seus primórdios e sempre ocupou um local privilegiado em todas as culturas, como elemento fundamental nos rituais religiosos, fonte de água não contaminada ou ainda presença constante nos momentos de comemoração e de confraternização, quando se brinda a todos e a tudo (1).

A dependência ao álcool é um grave problema de saúde pública no Brasil e no mundo. De acordo com dados publicados pela Organização Mundial da Saúde estima-se consumo per capita de 10,1 L de etanol para o ano de 2025, no Brasil. Estatisticamente é responsável por 3,2\% das mortes no mundo o que em dado absoluto, representa cerca de 5,5 milhões de morte/ano.

Seu tratamento é desafiador, mesmo para os melhores programas terapêuticos disponíveis. Esta dificuldade decorre do pequeno número de medicamentos aprovados para o uso e também pela elevada taxa de abandono, próximo a $50 \%(2,3)$. A naltrexona, administrada por via oral e atualmente por via intramuscular é uma das poucas opções farmacológicas eficazes disponíveis para o tratamento desta condição.

Sabemos que um dos grandes problemas para o tratamento de algumas doenças crônicas é a incidência de efeitos adversos e alta frequência de administração necessária para alguns fármacos, o que acaba levando à falta de adesão às recomendações médicas, um problema enfrentado no dia-a-dia pelos profissionais da saúde. Aqui propomos o desenvolvimento de sistemas biorresponsivos nanoestruturados para a liberação sustentada de cloridrato de naltrexona, utilizando a via subcutânea como via alternativa à administração oral da naltrexona a fim de melhorar a adesão ao tratamento farmacológico. 


\subsection{Alcoolismo e alternativas para tratamento}

O transtorno por uso do álcool representa um grande problema de saúde pública devido à sua prevalência, consequências e custos para sistemas de saúde e consequentemente para a sociedade (4). Em 1966 a Associação Médica Americana (AMA) passou a considerar o alcoolismo como doença e em 1988 incluiu as dependências a outras drogas como condições médicas passíveis de tratamento.

O Departamento Nacional de Saúde nos EUA publicou em abril de 2010 que 28\% dos adultos acima de 18 anos consomem álcool em níveis que os colocam em risco ao desenvolvimento do alcoolismo e problemas hepáticos, entre outros problemas médicos e psicossociais (5).

Uma pesquisa mais recente conduzida pelo Departamento de Saúde Americano (U.S. Department of Health and Human Services) disponível em 2012 apontou que 9.2\% da população com idade igual ou superior a 12 anos utilizaram drogas ilícitas durante o mês anterior à pesquisa (aproximadamente 30\% admitiram ter usado uma vez na vida) (6). Entre as drogas ilícitas, a maconha (cujo uso médico e até recreacional é legalizado em alguns estados americanos) é a mais utilizada, enquanto que o uso ilegal de analgésicos opióides (como fentanil e oxicodona) encontra-se em ascensão, o que é refletido na busca por tratamento $(6,7)$.

Aproximadamente 52\% destes indivíduos admitiram a ingestão de álcool, sendo que $6.2 \%$ costumam ingerir 5 doses ou mais uma vez por semana. Paralelamente, sabe-se que as lesões traumáticas representam cerca de $30 \%$ de vidas perdidas nos Estados Unidos anualmente entre pessoas de 1 a 44 anos. Em pacientes traumatizados na emergência, encontra-se a exposição ao álcool em cerca de $30 \%$ dos casos (7).

O panorama brasileiro não é muito diferente. De acordo com dados publicados do Observatório Brasileiro de Informações sobre Drogas do Ministério da Justiça em 2010, conclui-se que "álcool e tabaco são as drogas de maior prevalência de uso na vida, em todas as capitais brasileiras seguidas pelos inalantes". O levantamento realizado em 2007, pela Secretaria Nacional de Políticas Sobre Drogas (Senad) investigou os Padrões de Consumo de Álcool na População Brasileira. O estudo foi realizado em 143 municípios do País e detectou que 52\% dos brasileiros acima de 18 anos faz uso de bebida alcoólica pelo menos uma vez ao ano. Do conjunto dos homens adultos, $11 \%$ bebem todos os dias e $28 \%$ de 1 a 4 vezes por semana. Quanto à intensidade do consumo de bebidas alcoólicas, 24\% da população bebe frequentemente e pesado (pelo menos uma vez por semana, 5 ou mais 
doses) e $29 \%$ são bebedores pouco frequentes e não fazem uso "pesado". De acordo com dados do "National Institute on Alcohol Abuse and Alcholism" estadunidense cerca de 1,5 milhão de adultos receberam tratamento para alcoolismo em instalações especializadas em 2014.

O álcool é um depressor do sistema nervoso central (SNC) que produz inicialmente euforia, diminuição da ansiedade e excitação em concentração sérica baixa e resposta comportamental variável. $\mathrm{O}$ efeito deste está intimamente relacionado à concentração sérica; entre $0,01-0,05 \mathrm{gmL}^{-1}$ tem-se comportamento de aspecto normal, já concentração superior a $0,35 \mathrm{gmL}^{-1}$ confere estado de coma (8). Compreende desde uma intoxicação aguda, com sintomas que incluem sonolência, ataxia e fala arrastada, até torpor e coma (5). O indivíduo que consome bebida alcoólica de forma excessiva, ao longo do tempo, pode desenvolver dependência, condição conhecida como alcoolismo. Os fatores que podem levar ao alcoolismo são variados, envolvendo aspectos de origem biológica, psicológica e sociocultural. A maioria dos parâmetros de farmacocinética do álcool estão sujeitos à grande variabilidade intersubjetiva, como exemplificado pelas altas concentrações de álcool no sangue atingidas na ingestão de doses idênticas ajustadas em peso, tempo até o pico após o final do consumo e a taxa de eliminação de álcool do sangue (8). O etanol é metabolizado pela álcool desidrogenase com a produção de acetaldeído, que é então metabolizado por uma aldeído desidrogenase mitocondrial conhecida como ALDH2.

Quanto ao mecanismo de ação do etanol, as primeiras hipóteses eram voltadas para sua ação em membranas lipídicas. Pensava-se que, devido ao tamanho pequeno da molécula $\left(\mathrm{C}_{2} \mathrm{H}_{6} \mathrm{O}\right)$ e à natureza não específica da molécula de álcool, provavelmente não teria um domínio de ligação específico sobre as proteínas, mas interferiria nas membranas lipídicas da central sistema nervoso (SNC). No entanto, o etanol interage com a bicamada lipídica em concentrações muito superiores às clinicamente relevantes $(9,10)$. Desde então, os pesquisadores buscam outros alvos moleculares da droga. Os efeitos do álcool no encéfalo incluem mudanças nos níveis e função de neurotransmissores, canais iônicos, receptores, enzimas e outras moléculas intracelulares, culminando em mudanças sinápticas em circuitos cerebrais que regulam compulsividade e inibição de comportamento. As mudanças nestes sistemas moleculares levam à tolerância e abstinência, quando o álcool é removido do sistema. As ações primárias inibitórias e ativadoras do etanol sobre os diferentes canais e receptores iônicos dependem de uma 
série de variáveis, especialmente da concentração de álcool e a composição da subunidade de um canal ou receptor particular (11).

As regiões cerebrais que desempenham um papel importante na mediação dos efeitos reforçadores do etanol foram identificadas por uma variedade de estudos neurofarmacológicos, com lesão de regiões específicas, microinjeção e microdiálise. A amígdala estendida e a via dorsal mesolímbica, incluindo a área tegmental ventral do mesencéfalo (VTA), o nucleo accumbens e o córtex pré-frontal, são as principais regiões encefálicas que medeiam as ações reforçadoras do álcool $(12,13)$. A via dopaminérgica mesolímbica é conhecida por ser controlada, em grande parte, pela atividade glutamatérgica na VTA. O sistema serotoninérgico proveniente do núcleo dorsal da rafe também exerce influência na atividade dopaminérifica da VTA e do núcleo accumbens. A VTA também é regulado pelo GABA e projeções encefalinérgicas do pálido ventral e do núcleo accumbens. Finalmente, os cannabinoides (CBs) e os opióides podem aumentar a liberação de dopamina no núcleo accumbens, ativando os neurônios dopaminérgicos na VTA $(11,14,15)$

A via mesolímbica da dopamina para a VTA e para o nucleo accumbens / e o estriado ventral (NcAcc/VS) possuem um importante papel no reforço positivo do álcool (16). Os níveis extracelulares de dopamina nos roedores no nucleo accumbens estão aumentados tanto quando se faz a injeção deste, ou quando da sua administração por via oral (17). O álcool induz a liberação de dopamina no nucleo accumbens / e o estriado ventral e parece mediar a ativação de dopamina nos neurônios no VTA; esse aumento da taxa de disparo é visto tanto in vivo quanto in vitro (16). A administração do etanol como primeiro efeito, ocasionaria mudanças nos corpos celulares dopaminérgicos, localizados na VTA como aumento de liberação de dopamina no NcAcc, logo maior estimulação de receptores D1 e D2, pelo neurotransmissor conferindo aumento da atividade locomotora (via pálio ventral) e efeito reforçador do etanol.

Estudos com roedores têm demostrado que o aumento de dopamina no NcAcc após a administração aguda de álcool ocorre, em parte, por uma indução da liberação endógena de opióide, um achado confirmado em humanos $(18,19)$. O reforço positivo do álcool resulta de uma interação entre álcool, opióides endógenos e dopamina, sendo mediado em parte pela liberação endógena de opióides na área ventral tegumentar do mesencéfalo. Estes opióides inibem a ação inibitória de interneurônios GABAérgicos (ácido gama-aminobutírico), desinibindo a via dopaminérgica. Uma vez essa via "desinibida", aumentam os disparos neuronais, aumentando a liberação de dopamina no nucleo 
accumbens (20) (figura 1). Assim sendo, o receptor $\mu$-opióide parece ter importância particular no reforço positivo do álcool.

Assim, os opióides endógenos estão envolvidos não só nas ações das drogas classificadas como opióides, como também nos efeitos do álcool. Existem três classes de opióides endógenos: endorfinas, encefalinas e dinorfinas. Todos exercem seus efeitos ao interagir com três subtipos de receptores opióides: $\mu, \delta$ e $\mathrm{K}$. A hipótese de que o reforço positivo do álcool é mediado, pelo menos em parte, pela liberação de opióides endógenos no encéfalo é apoiada por numerosos estudos que demonstram que os antagonistas de opióides suprimem o consumo de álcool em uma variedade de espécies e modelos (21). Além disso, a inativação completa (isto é, knockout) do receptor de opióides $\mu$ bloqueia a auto-administração de álcool em camundongos. A naltrexona, um antagonista de receptor de opiáceos subtipo-inespecífico, atualmente aprovado como um tratamento para o alcoolismo em seres humanos é particularmente eficaz na redução do consumo excessivo de álcool. Os sistemas opióides influenciam as ações do etanol tanto através da interação com o sistema dopaminérgico mesolímbico, quanto independente dele, como demonstrado pelos aumento induzido pelo etanol no conteúdo extracelular de endorfina no nucleo accumbens (ver figura 1).

Os antagonistas dos receptores de opióides interferem com os efeitos recompensadores do álcool, atuando em locais na área ventral tegmental, nucleo accumbens e núcleo central da amígdala (22).

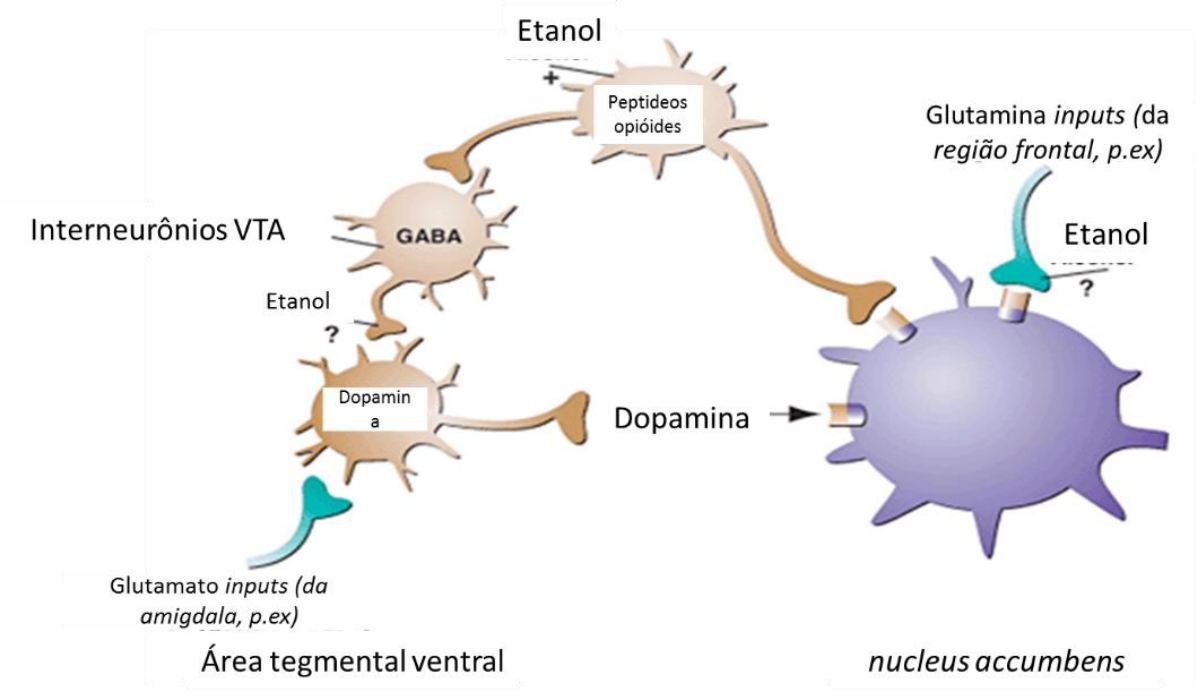

Figura 1- Modulação da via dopaminérgica mesolímbica pelo sistema opioide. Fonte: National Institute on Alcohol Abuse and Alcoholism (NIAAA). 
As propriedades reforçadoras do álcool podem ser avaliadas usando um procedimento chamado preferência condicionamento por lugar. O condicionamento é amplamente definido como um pareamento entre um estímulo não-condicionado e um estímulo condicionado. $O$ estímulo não-condicionado é a administração do fármaco ou outra recompensa ao modelo, e o estimulo condicionado é o ambiente distinto no qual o animal é colocado após a administração do fármaco ou recompensa (23). O pareamento muitas vezes se desenvolve de uma associação entre os estímulos não-condicionado e o estimulo condicionado que resulta em uma futura resposta condicionada (CR). O CR pode assumir a forma de preferência ou aversão exibida durante o período de teste. No período de teste, o organismo tem acesso livre para entrar em uma câmara previamente associada ao medicamento ou para entrar em uma câmara não associada ao medicamento (24). Neste procedimento, as pistas contextuais estão associadas à presença ou ausência da droga por exemplo, os roedores são repetidamente colocados em um ambiente específico onde recebem etanol e em outro ambiente onde recebem salina. Posteriormente, os roedores podem explorar livremente esses ambientes. Se o animal gasta mais tempo explorando o ambiente pareado com etanol, esse comportamento reflete os efeitos de reforço positivo condicionado do etanol (25).

\subsection{Naltrexona e outros fármacos utilizados no tratamento da dependência ao álcool}

O fármaco naltrexona foi aprovado pela agência regulatória americana Food and Drug Administration (FDA) como farmacoterapia para o alcoolismo e sua eficácia tem suporte em uma meta análise de muitos estudos clínicos randomizados (26). As mensurações motivacionais descritas foram suprimidas pela deleção genética do receptor $\mu$-opióide nos ratos, e resultado similar foi obtido com antagonistas $\mu$-opióides. (16, 27, 28). Outros fármacos aprovados incluem o acamprosato, que age nos neurotramissores de GABA e glutamato, o topiramato, que exerce seu efeito aumentando a inibição GABA e reduzindo a neurotransmissão glutamatérgica, e o dissulfiram, que interfere na degradação do álcool, resultando no acúmulo de acetaldeído, o que produz uma reação de "desprazer" (náuseas, palpitações, rubor facial, por exemplo).

A naltrexona é um antagonista de opióides com maior afinidade aos receptores do tipo $\mu$, de maneira que o paciente não obtém a sensação de prazer/ euforia associada ao uso de opióides $(10,11)$. A naltrexona está relacionada quimicamente com outro antagonista de receptores opióides conhecido como naloxona, entretanto sua 
biodisponibilidade oral e a duração de ação são maiores (16). Na figura 2, é representada a estrutura molecular do fármaco.

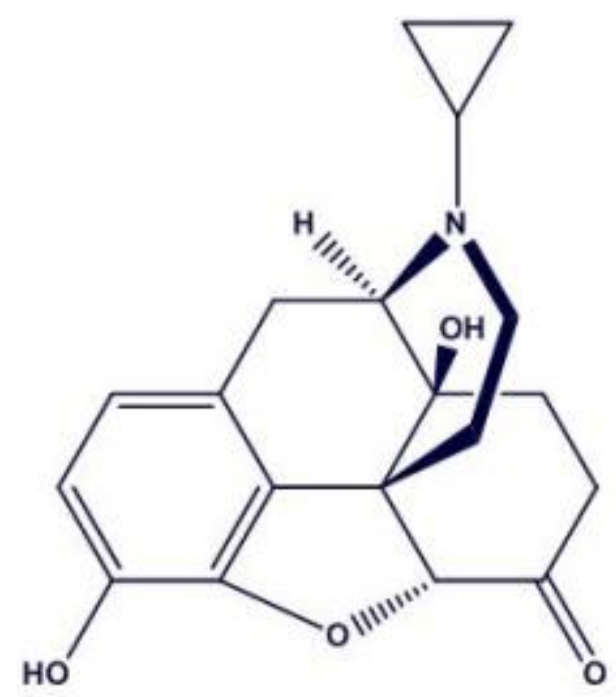

Figura 2- Representação da estrutura química da molécula de naltrexona.

O fármaco também reduz a taxa de recaída do consumo e o desejo compulsivo pelo álcool (29). Há ainda relatos de melhora terapêutica quando da associação de naltrexona e terapia cognitiva comportamental (30). Além de sua utilização no tratamento de dependência ao álcool, sua administração é também aprovada pelo FDA para o tratamento da dependência a opióides, além de ter se mostrado eficaz (mas ainda sem ser aprovado) no tratamento de dependência a metanfetamina e cocaína (em associação à buprenorfina) e no tratamento de compulsão por jogos e obesidade (em associação com bupropiona) (30).

A despeito de suas múltiplas potenciais aplicações, o uso oral da naltrexona é limitado pela sua extensiva eliminação pré-sistemica o que resulta em biodisponibilidade baixa que varia entre 5 e 40\%, e necessidade de administração frequente $(31,32)$. De frente a este enunciado, sistemas de liberação que utilizem via de administração diferente da oral e ao mesmo tempo, apresentem a propriedade de prolongar a liberação deste fármaco, podem permitir contornar o efeito do metabolismo de primeira passagem além de reduzir a frequência de administração melhorando a adesão ao tratamento. 


\subsection{Liberação sustentada e sistemas de liberação líquido-cristalinos}

Diversos sistemas de liberação têm sido investigados para a liberação modificada de naltrexona, incluindo micropartículas, géis, dispositivos transdérmicos, comprimidos bucais e implantes subcutâneos (33-37). De fato, o interesse por novas formulações que contornem as desvantagens apontadas está bem ilustrado em revisões recentemente publicadas $(31,38-40)$.

Dentre estes, micropartículas para administração intramuscular e implantes poliméricos subcutâneos estão em fase de estudo clínico (implantes) ou receberam aprovação (micropartículas) em alguns países para o tratamento de alcoolismo e/ou dependência associada a opióides. Apesar de seus benefícios, tanto as micropartículas quanto os implantes oferecem desvantagens significativas. Desvantagens associadas às micropartículas incluem o fato do processo de produção ser geralmente dispendioso, envolver múltiplos passos e equipamentos específicos. Além disso, sua administração intramuscular (via aprovada para o sistema) requer um profissional treinado. Já os implantes necessitam de procedimentos cirúrgicos para sua inserção e, com frequência, retirada $(29,41)$. Com base nesses problemas, faz-se necessário o desenvolvimento de novas formas farmacêuticas que permitam a liberação prolongada do fármaco por algumas semanas e uma administração mais fácil.

Para tal, propomos o uso de formulações precursoras fluidas, mais especificamente microemulsões que, mediante administração subcutânea e absorção de fluidos do tecido, sofram geleificação in vivo formando sistemas líquido-cristalinos nanoestruturados. Com isso propomos combinar a simplicidade da obtenção e estabilidade termodinâmica de microemulsões, a disponibilidade de tensoativos relativamente baratos para sua obtenção, e a habilidade de sistemas líquido-cristalinos de sustentar e modular a liberação de compostos. Conforme mostra a figura 3, a formulação precursora (microemulsão), após administração subcutânea deve absorver água do tecido subcutâneo (processo de geleificação), formando assim um sistema nanoestruturado com habilidade de sustentar a liberação do fármaco. 


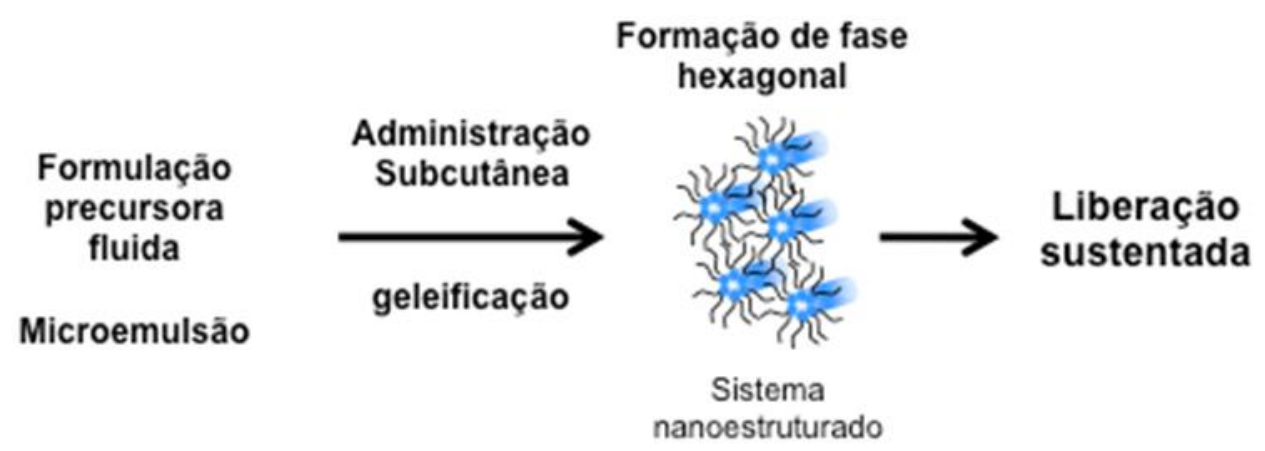

Figura 3- Esquema do sistema proposto e formação do gel in situ.

As microemulsões (ME) são agregados, muitas vezes esféricos, com diâmetro geralmente inferior a 150nm, tipicamente da ordem de $100 \mathrm{~nm}(42,43)$. Apesar da denominação "micro", o sistema é formado por gotículas com tamanhos suficientemente pequenos (na faixa nanométrica) para ser opticamente transparente. Por essa razão, outras denominações, como "submicron emulsion" têm sido utilizadas para designar esse sistema. ME são, de forma geral, definidas como sistemas termodinamicamente estáveis, isotrópicos e transparentes, resultantes da mistura de dois líquidos imiscíveis, (usualmente água e óleo) estabilizados por um filme de compostos tensoativos, localizados na interface óleo/água (42-44).

Os sistemas líquido-cristalinos são frequentemente encontrados em nossas vidas. Por exemplo, as membranas das células são resultado de uma fase líquido-cristalina liotrópica que é originada pela agregação de fosfolipides em água. Os cristais líquidos podem ser definidos como o estado da matéria cujas propriedades mecânicas e simétricas são intermediárias entre os sólidos cristalinos e os líquidos isotrópicos. Nos cristais, as moléculas estão altamente organizadas enquanto que nos líquidos, as moléculas difundemse livremente (45). Sistemas líquido-cristalinos combinam a organização de moléculas dos cristais sólidos com o movimento das moléculas no estado líquido. Os sistemas líquidocristalinos são de grande importância para administração de fármacos, pois podem incorporar compostos com solubilidades variadas, proteger os fármacos da degradação física e enzimática, diminuir o efeito de primeira passagem e controlar sua liberação (4648).

São frequentemente classificados em sistemas termotrópicos ou liotrópicos. 0 primeiro é obtido por aumento de temperatura e o liotrópico, por sua vez é influenciado pela 
proporção dos solventes. As fases mais comumente encontradas em sistemas liotrópicos são lamelar, hexagonal e cúbica (49), conforme ilustrado na figura 4.

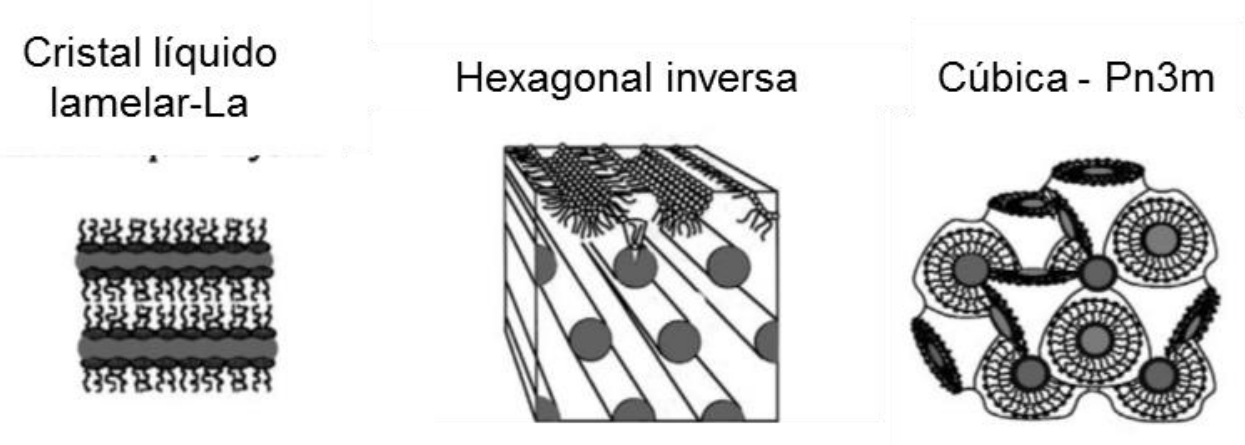

Figura 4- Representação esquemática de fases líquido-cristalinas. Adaptado de Spomenka Milak e Andreas Zimmer (49).

A fase lamelar consiste em estruturas lineares de bicamadas lipídicas alternadas entre canais de água e pode ser usada para obter a fase cúbica por sua habilidade de absorver excesso de água dos fluidos biológicos (49).

A fase líquido-cristalina cúbica exibe a mais complexa organização espacial conhecida das fases liquido-cristalinas. O nome "cúbico" originou-se a partir de resultados de difração de raios -X que mostraram simetria cúbica (49). A estrutura da fase cúbica consiste em bicamadas curvas, que se estendem em três dimensões, apresentam espessura estimada de 3,5 $\mathrm{nm}$ e separam duas redes de canais de água contínuos. Esses canais não se intereceptam, e exibem diâmetro de aproximadamente $5 \mathrm{~nm}$ quando completamente intumescidos (50).

A fase hexagonal é caracterizada por longas estruturas cilíndricas bidimensionais, podendo existir na forma reversa, na qual as cabeças polares do composto formador de fase ficam na região interna dos cilindros e a porção apolar fica localizada ao redor dos cilindros; ou na normal, onde as cabeças polares do composto localizam-se na região externa dos cilindros $(51,52)$. Embora essas fases se apresentem macroscopicamente como géis, a viscosidade é bastante diferente nas fases cúbica, hexagonal e lamelar, o que pode influenciar a liberação do fármaco (49).

A fase hexagonal foi selecionada aqui em decorrência da sua estrutura nanométrica (diâmetro dos cilindros e espessura das bicamadas é da ordem de nanômetros) e versatilidade estrutural, caracterizada pela presença de domínios hidrofílicos e hidrofóbicos, apresentando a capacidade de prolongar a liberação dos compostos hidrofílicos com 
característica físico-químicas semelhantes ao cloridrato de naltrexona $(49,53)$. Além disso, ela apresenta viscosidade intermediária entre a cúbica e lamelar. Sabe-se que a viscosidade é de grande importância para a liberação de compostos; em decorrência de sua elevada viscosidade, a fase cúbica poderia resultar em liberação mais lenta do fármaco $(54,55)$.

A transição de uma fase menos viscosa como por exemplo uma microemulsão ou a fase lamelar em estrutura bicontínua cubica ou fase hexagonal reversa pode ocorrer em decorrência de alterações na temperatura, concentração de solvente (como água) e/ou adição de outros compostos, como fármacos $(48,53)$. Aqui propusemos sistemas capazes de se transformar em fase hexagonal (reversa e normal, dependendo do composto formador) mediante absorção de água. Essa transição pode ser explicada pelo fator de empacotamento $(K)$, que relaciona a forma da molécula do composto formador com propriedades que influenciam a curvatura da interface polar-apolar. $O$ fator de empacotamento representa a medida da geometria preferencialmente adotada pelo tensoativo quando da formação de agregados (56). Em outras palavras, é um parâmetro muito útil para predizer a mesofase que é preferencialmente formada por um composto, e pode ser calculada pela equação 1:

\title{
$\mathrm{K}=\mathrm{Vh} / \mathbf{A o} . \mathbf{L c}$ (equação 1)
}

\author{
$\mathbf{K}=$ fator de empacotamento \\ $\mathrm{Vh}=$ volume da parte hidrofóbica do tensoativo \\ $\mathbf{A o}=$ =área da secção transversal da cabeça polar do tensoativo \\ LC=comprimento da cauda hidrofóbica do tensoativo
}

De acordo com Engströn et.al, 1992 tem-se geralmente $\mathrm{k}$ 1 para a fase lamelar, 1,3 para fase cúbica bicontínua e $\sim 1,7$ fase hexagonal reversa. A transição entre fases em função do formato de compostos formadores de fase (principalmente a monoleína) e seu fator de empacotamento foi estudada e idealizada por diversos autores, conforme ilustrado na figura 5 (57): 
Fase invertida

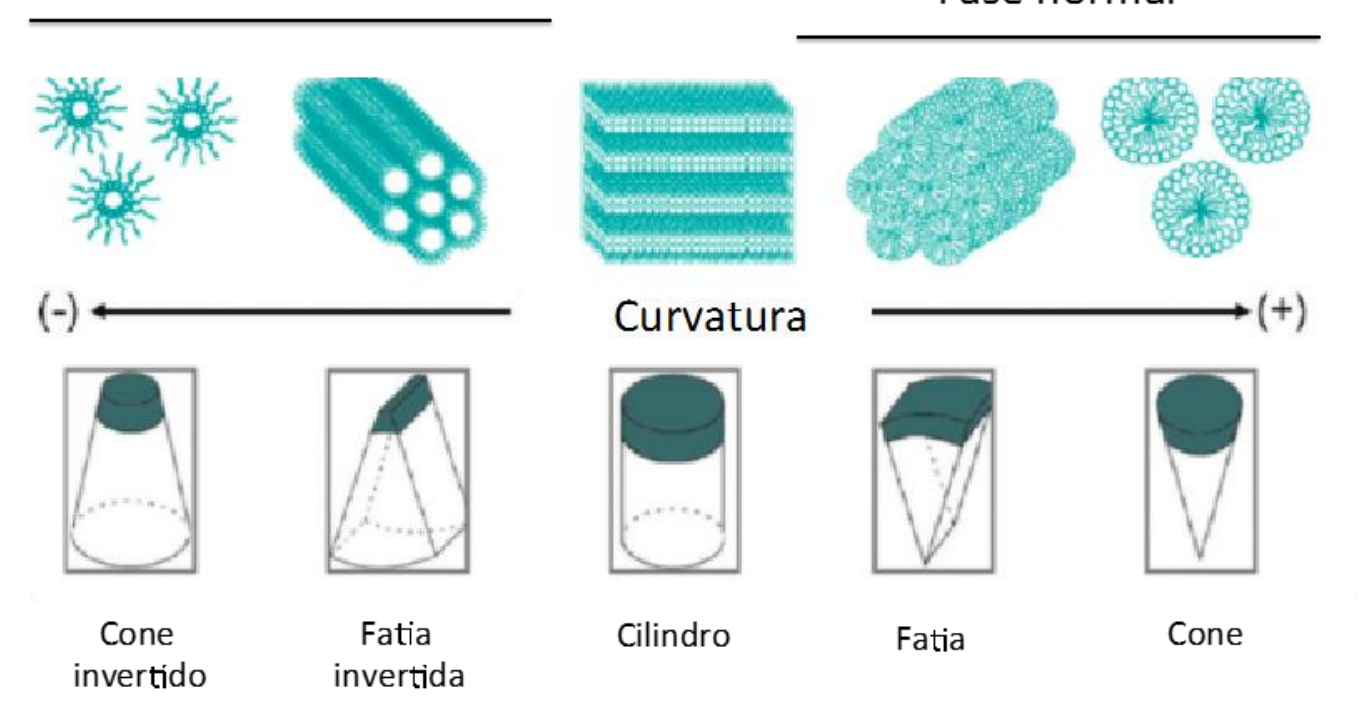

Figura 5- Idealização das fases formadas por tensoativos e outros lipídios formadores de fases em função do formato do tensoativo e seu fator de empacotamento. Adaptado de Kulkarni, Langmuir, 2011(57).

Para compostos que formam sistemas reversos como o monoglicerídeo monoleína, o aumento de água permite que os grupos das cabeças polares do monoglicerídeo se movam mais livremente em uma direção perpendicular relativa ao plano da camada de água. Estes movimentos promovem desordem da cadeia hidrofóbica do monoglicerídeo, aumentando assim Vh. Como a interação dos grupos da cabeça polar são fortes (em decorrência de ligações de hidrogênio), a sua seção transversal tende a ser constante, e o fator de empacotamento aumenta porque Vh aumenta, enquanto Ao e LC permanecem constantes, facilitando a transformação de fase lamelar para cúbica (47). Uma base racional semelhante pode ser utilizada para transformação da microemulsão $\mathrm{A} / \mathrm{O}$ em fase hexagonal reversa, já que estas são sistemas mais semelhantes aos micelares reversos (alguns autores utilizam o termo "micela intumescida" para microemulsões), sendo que o aumento de volume da cadeia apolar do tensoativos pode levar a formação do sistema hexagonal.

A transição entre fases quando do uso de compostos que formem sistemas normais na presença de água (como tensoativos BRIJ e Vitamina E TPGS) é menos estudada. Para vitamina E TPGS, por exemplo, acredita-se que a sequência de fases formada à medida que $o$ teor aquoso aumente seja: fase micelar reversa, fase hexagonal e fase micelar normal.(58) 
Comparado a outros sistemas de liberação sustentada desenvolvidos para naltrexona, o uso de microemulsões e sua geleificação com formação in vivo da fase hexagonal ou lamelar oferece diversas vantagens: $(28,29,39,41,59,60)$

- a viscosidade da formulação precursora pode ser ajustada com a fase oleosa a fim de facilitar sua administração subcutânea,

- microemulsões são de fácil preparação, o que reduz custos e complexidade relacionados ao desenvolvimento,

- a via subcutânea parece ser superior se comparada a outras vias no que diz respeito a potência da naltrexona em reduzir o consumo de etanol,

- a administração subcutânea é mais simples e não requer treinamento extensivo, de modo que familiares do paciente (se não o próprio) podem executar a administração (de maneira similar à insulina);

- ao contrário de implantes que devem permanecer no mesmo sítio, o local de administração do sistema proposto pode ser alternado a fim de reduzir possíveis reações locais. 


\section{CONCLUSÕES}

Microemulsões com diâmetro da fase dispersa inferior a 150nm foram obtidas com êxito utilizando diferentes tensoativos e co-tensoativos. As microemulsões ME-TPGS e MEMO originaram fase hexagonal mediante absorção de água, enquanto que ME-Span originou fase lamelar.

As microemulsões apresentam comportamento reológico tipo newtoniano e as fases hexagonal e lamelar compartam-se como fluido pseudoplástico. Todas as microemulsões foram capazes de incorporar o fármaco, embora em diferentes concentrações: a formulação ME-Span incorporou 1,3\%, enquanto que ME-TPGS e ME-MO incorporaram até $5 \%$. Independentemente da quantidade incorporada e do tipo de fase, a liberação pode ser melhor descrita de acordo com cinética de pseudo-primeira ordem. Essas formulações sustentaram a liberação do fármaco por tempo superior a 80 horas.

A microemulsão ME-MO formou gel de estrutura hexagonal após $48 \mathrm{~h}$, que se manteve por mais de 30 dias. Através da experimentação IVIS, observou-se fluorescência do marcador no local da aplicação por até 34 dias com a administração do gel, o que implica em liberação lenta, ao contrário do que foi observada quando da administração da solução do marcador.

A microemulsão ME-MO com $5 \%$ de naltrexona reduziu os efeitos recompensadores do etanol.

A microemulsão ME-MO com $10 \%$ de naltrexona antagonizou a preferência condicionada por lugar induzida pelo etanol. 


\section{REFERÊNCIAS*}

1. Gigliotti A, Bessa MA. [Alcohol Dependence Syndrome: diagnostic criteria]. Rev Bras Psiquiatr. 2004;26 Suppl 1:S11-3.

2. Haighton C, Wilson G, Ling J, McCabe K, Crosland A, Kaner E. A Qualitative Study of Service Provision for Alcohol Related Health Issues in Mid to Later Life. PLoS One. 2016;11(2):e0148601.

3. Valencia JG, Méndez Villanueva MP. [Factors Associated With the Temporary Abandonment of Treatment for Disorders Due to Substance Abuse in an Institution in Medellin, Colombia]. Rev Colomb Psiquiatr. 2014;43(1):7-17.

4. Dupuy M, Chanraud S. Imaging the Addicted Brain: Alcohol. Int Rev Neurobiol. 2016;129:1-31.

5. Costin BN, Miles MF. Molecular and neurologic responses to chronic alcohol use. Handb Clin Neurol. 2014;125:157-71.

6. Substance Abuse and Mental Health Services Administration, Results from the 2012 National Survey on Drug Use and Health: Summary of National Findings, NSDUH Series H46, HHS Publication No. (SMA) 13-4795. Rockville, MD: Substance Abuse and Mental Health Services Administration. [Internet]. 2013.

7. Afshar M, Richards S, Mann D, Cross A, Smith GB, Netzer G, et al. Acute immunomodulatory effects of binge alcohol ingestion. Alcohol. 2015;49(1):57-64.

8. Dubowski KM. Absorption, distribution and elimination of alcohol: highway safety aspects. J Stud Alcohol Suppl. 1985;10:98-108.

9. Goldstein MS, Surber M, Wilner DM. Outcome evaluations in substance abuse: a comparison of alcoholism, drug abuse, and other mental health interventions. Int $\mathrm{J}$ Addict. 1984;19(5):479-502.

10. Peoples RW, Li C, Weight FF. Lipid vs protein theories of alcohol action in the nervous system. Annu Rev Pharmacol Toxicol. 1996;36:185-201.

11. Vengeliene V, Bilbao A, Molander A, Spanagel R. Neuropharmacology of alcohol addiction. Br J Pharmacol. 2008;154(2):299-315.

12. Volkow ND, Koob GF, McLellan AT. Neurobiologic Advances from the Brain Disease Model of Addiction. N Engl J Med. 2016;374(4):363-71.

\footnotetext{
* De acordo com International Committee of Medical Journal Editors. [Internet]. Uniform requirements for manusripts submitted to Biomedial Journals. [2011 jul 15]. Available from: http://www.nlm.nih.gov/bsd/uniform_requirements.html.
} 
13. Koob GF, Volkow ND. Neurobiology of addiction: a neurocircuitry analysis. Lancet Psychiatry. 2016;3(8):760-73.

14. Spanagel R, Zieglgänsberger W. Anti-craving compounds for ethanol: new pharmacological tools to study addictive processes. Trends Pharmacol Sci. 1997;18(2):549 .

15. Katner SN, Magalong JG, Weiss F. Reinstatement of alcohol-seeking behavior by drug-associated discriminative stimuli after prolonged extinction in the rat. Neuropsychopharmacology. 1999;20(5):471-9.

16. Spagnolo PA, Ramchandani VA, Schwandt ML, Zhang L, Blaine SK, Usala JM, et al. Effects of naltrexone on neural and subjective response to alcohol in treatment-seeking alcohol-dependent patients. Alcohol Clin Exp Res. 2014;38(12):3024-32.

17. Acquas E, Meloni M, Di Chiara G. Blockade of delta-opioid receptors in the nucleus accumbens prevents ethanol-induced stimulation of dopamine release. Eur $\mathrm{J}$ Pharmacol. 1993;230(2):239-41.

18. Mitchell JM, Tavares VC, Fields HL, D'Esposito M, Boettiger CA. Endogenous opioid blockade and impulsive responding in alcoholics and healthy controls. Neuropsychopharmacology. 2007;32(2):439-49.

19. Mitchell JM, Bergren LJ, Chen KS, Rowbotham MC, Fields HL. Naltrexone aversion and treatment efficacy are greatest in humans and rats that actively consume high levels of alcohol. Neurobiol Dis. 2009;33(1):72-80.

20. Brett J, Ivers R, Doyle M, Lawrence L, Conigrave K. Should naltrexone be the firstline medicine to treat alcohol dependence in Aboriginal and Torres Strait Islander populations? An Australian perspective. Aust Fam Physician. 2015;44(11):815-9.

21. Ulm RR, Volpicelli JR, Volpicelli LA. Opiates and alcohol self-administration in animals. J Clin Psychiatry. 1995;56 Suppl 7:5-14.

22. Koob GF. Alcoholism: allostasis and beyond. Alcohol Clin Exp Res. 2003;27(2):23243.

23. Tzschentke TM. Measuring reward with the conditioned place preference (CPP) paradigm: update of the last decade. Addict Biol. 2007;12(3-4):227-462.

24. Lucke-Wold B. The Varied Uses of Conditioned Place Preference in Behavioral Neuroscience Research: An Investigation of Alcohol Administration in Model Organisms. Impulse (Columbia). 2011;2011. 
25. Cunningham $\mathrm{CL}$, Henderson CM, Bormann NM. Extinction of ethanol-induced conditioned place preference and conditioned place aversion: effects of naloxone. Psychopharmacology (Berl). 1998;139(1-2):62-70.

26. Johnson BA, Ait-Daoud N, Prihoda TJ. Combining ondansetron and naltrexone effectively treats biologically predisposed alcoholics: from hypotheses to preliminary clinical evidence. Alcohol Clin Exp Res. 2000;24(5):737-42.

27. Egli M. Can experimental paradigms and animal models be used to discover clinically effective medications for alcoholism? Addict Biol. 2005;10(4):309-19.

28. Heilig M, Egli M. Pharmacological treatment of alcohol dependence: target symptoms and target mechanisms. Pharmacol Ther. 2006;111(3):855-76.

29. Johnson BA. Naltrexone long-acting formulation in the treatment of alcohol dependence. Ther Clin Risk Manag. 2007;3(5):741-9.

30. Anton RF, Kranzler HR, Meyer RE. Neurobehavioral aspects of the pharmacotherapy of alcohol dependence. Clin Neurosci. 1995;3(3):145-54.

31. Lobmaier PP, Kunoe N, Gossop M, Waal H. Naltrexone depot formulations for opioid and alcohol dependence: a systematic review. CNS neuroscience \& therapeutics. 2011;17(6):629-36.

32. Anton RF, Moak DH, Waid LR, Latham PK, Malcolm RJ, Dias JK. Naltrexone and cognitive behavioral therapy for the treatment of outpatient alcoholics: results of a placebocontrolled trial. Am J Psychiatry. 1999;156(11):1758-64.

33. Yin W, Akala EO, Taylor RE. Design of naltrexone-loaded hydrolyzable crosslinked nanoparticles. Int J Pharm. 2002;244(1-2):9-19.

34. Ngo HT, Arnold-Reed DE, Hansson RC, Tait RJ, Hulse GK. Blood naltrexone levels over time following naltrexone implant. Prog Neuropsychopharmacol Biol Psychiatry. 2008;32(1):23-8.

35. Giannola LI, De Caro V, Giandalia G, Siragusa MG, Tripodo C, Florena AM, et al. Release of naltrexone on buccal mucosa: permeation studies, histological aspects and matrix system design. Eur J Pharm Biopharm. 2007;67(2):425-33.

36. Milewski M, Stinchcomb AL. Vehicle composition influence on the microneedleenhanced transdermal flux of naltrexone hydrochloride. Pharm Res. 2011;28(1):124-34.

37. Kjoniksen AL, Calejo MT, Zhu K, Cardoso AM, de Lima MC, Jurado AS, et al. Sustained release of naltrexone from poly(n-isopropylacrylamide) microgels. J Pharm Sci. 2014;103(1):227-34. 
38. Goonoo N, Bhaw-Luximon A, Ujoodha R, Jhugroo A, Hulse GK, Jhurry D. Naltrexone: A review of existing sustained drug delivery systems and emerging nano-based systems. $J$ Control Release. 2014;183C:154-66.

39. Hulse GK. Improving clinical outcomes for naltrexone as a management of problem alcohol use. Br J Clin Pharmacol. 2013;76(5):632-41.

40. Krupitsky EM, Blokhina EA. Long-acting depot formulations of naltrexone for heroin dependence: a review. Current opinion in psychiatry. 2010;23(3):210-4.

41. Hulse GK, Stalenberg V, McCallum D, Smit W, O'Neil G, Morris N, et al. Histological changes over time around the site of sustained release naltrexone-poly(DL-lactide) implants in humans. J Control Release. 2005;108(1):43-55.

42. Lawrence MJ, Rees GD. Microemulsion-based media as novel drug delivery systems. Adv Drug Deliv Rev. 2000;45(1):89-121.

43. Lopes LB. Overcoming the cutaneous barrier with microemulsions. Pharmaceutics. 2014;6(1):52-77.

44. Kreilgaard M. Influence of microemulsions on cutaneous drug delivery. Adv Drug Deliv Rev. 2002;54 Suppl 1:S77-98.

45. Singh AN, Srivastava S, Jainar AK. Pharmacotherapy of chronic alcoholism: a review. Drugs Today (Barc). 1999;35(1):27-33.

46. Kogan A, Shalev DE, Raviv U, Aserin A, Garti N. Formation and characterization of ordered bicontinuous microemulsions. J Phys Chem B. 2009;113(31):10669-78.

47. Lopes LB, Brophy CM, Furnish E, Flynn CR, Sparks O, Komalavilas P, et al. Comparative study of the skin penetration of protein transduction domains and a conjugated peptide. Pharm Res. 2005;22(5):750-7.

48. Phelps J, Bentley MV, Lopes LB. In situ gelling hexagonal phases for sustained release of an anti-addiction drug. Colloids Surf B Biointerfaces. 2011;87(2):391-8.

49. Milak S, Zimmer A. Glycerol monooleate liquid crystalline phases used in drug delivery systems. Int J Pharm. 2015;478(2):569-87.

50. Guo C, Wang J, Cao F, Lee RJ, Zhai G. Lyotropic liquid crystal systems in drug delivery. Drug Discovery Today. 2010;15(23-24):1032-40.

51. Cremonez CP, Bentley MVLB, Lara MG. Caracterização do intumescimento de sistemas líquido-cristalinos de fase lamelar de monoleína e água contendo polihexametilenobiguanida. Rev Ciênc Farm Básica Apl: 2013;34(4):545-553. 
52. Boyd BJ, Whittaker DV, Khoo S-M, Davey G. Lyotropic liquid crystalline phases formed from glycerate surfactants as sustained release drug delivery systems. International Journal of Pharmaceutics. 2006;309(1-2):218-26.

53. Hosmer JM, Shin SH, Nornoo A, Zheng H, Lopes LB. Influence of internal structure and composition of liquid crystalline phases on topical delivery of paclitaxel. J Pharm Sci. 2011;100(4):1444-55.

54. Kiefer F, Jahn H, Otte C, Demiralay C, Wolf K, Wiedemann K. Increasing leptin precedes craving and relapse during pharmacological abstinence maintenance treatment of alcoholism. J Psychiatr Res. 2005;39(5):545-51.

55. Otte A, Báez-Santos YM, Mun EA, Soh BK, Lee YN, Park K. The in vivo transformation and pharmacokinetic properties of a liquid crystalline drug delivery system. Int J Pharm. 2017.

56. Aboofazeli R, Barlow DJ, Lawrence MJ. Particle size analysis of concentrated phospholipid microemulsions I. Total intensity light scattering. AAPS PharmSci. 2000;2(2):E13.

57. Kulkarni CV. Nanostructural studies on monoelaidin-water systems at low temperatures. Langmuir. 2011;27(19):11790-800.

58. Guo Y, Luo J, Tan S, Otieno BO, Zhang Z. The applications of Vitamin E TPGS in drug delivery. Eur J Pharm Sci. 2013;49(2):175-86.

59. Roozen $H G$, de Waart $R$, van den Brink W. Efficacy and tolerability of naltrexone in the treatment of alcohol dependence: oral versus injectable delivery. Eur Addict Res. 2007;13(4):201-6.

60. Williams KL, Broadbridge CL. Potency of naltrexone to reduce ethanol selfadministration in rats is greater for subcutaneous versus intraperitoneal injection. Alcohol. 2009;43(2):119-26.

61. Hosmer JM, Steiner AA, Lopes LB. Lamellar liquid crystalline phases for cutaneous delivery of Paclitaxel: impact of the monoglyceride. Pharm Res. 2013;30(3):694-706.

62. Pepe D, McCall M, Zheng H, Lopes LB. Protein transduction domain-containing microemulsions as cutaneous delivery systems for an anticancer agent. J Pharm Sci. 2013;102(5):1476-87.

63. Pepe D, Phelps J, Lewis K, Dujack J, Scarlett K, Jahan S, et al. Decylglucoside-based microemulsions for cutaneous localization of lycopene and ascorbic acid. Int $\mathrm{J}$ Pharm. 2012;434(1-2):420-8. 
64. Vicentini FT, Vaz MM, Fonseca YM, Bentley MV, Fonseca MJ. Characterization and stability study of a water-in-oil microemulsion incorporating quercetin. Drug Dev Ind Pharm. 2011;37(1):47-55.

65. Rosenholm JB. Phase equilibriums, self-assembly and interactions in two-, three- and four medium-chain length component systems. Adv Colloid Interface Sci. 2014;205:9-47.

66. Garti N, Yaghmur A, Leser ME, Clement V, Watzke HJ. Improved oil solubilization in oil/water food grade microemulsions in the presence of polyols and ethanol. J Agric Food Chem. 2001;49(5):2552-62.

67. Moghimipour E, Salimi A, Eftekhari S. Design and characterization of microemulsion systems for naproxen. Adv Pharm Bull. 2013;3(1):63-71.

68. Moghimipour E, Salimi A, Leis F. Preparation and evaluation of tretinoin microemulsion based on pseudo-ternary phase diagram. Adv Pharm Bull. 2012;2(2):141-7. 69. Moghimipour E, Salimi A, Karami M, Isazadeh S. Preparation and characterization of dexamethasone microemulsion based on pseudoternary phase diagram. Jundishapur J Nat Pharm Prod. 2013;8(3):105-12.

70. Kawakami K, Yoshikawa T, Hayashi T, Nishihara Y, Masuda K. Microemulsion formulation for enhanced absorption of poorly soluble drugs. II. In vivo study. J Control Release. 2002;81(1-2):75-82.

71. Yaghmur A, Rappolt M, Østergaard J, Larsen C, Larsen SW. Characterization of bupivacaine-loaded formulations based on liquid crystalline phases and microemulsions: the effect of lipid composition. Langmuir. 2012;28(5):2881-9.

72. Khodaverdi E, Tekie FS, Mohajeri SA, Ganji F, Zohuri G, Hadizadeh F. Preparation and investigation of sustained drug delivery systems using an injectable, thermosensitive, in situ forming hydrogel composed of PLGA-PEG-PLGA. AAPS PharmSciTech. 2012;13(2):590-600.

73. Zhang R, Wood AL, Enfield CG, Jeong SW. Stochastical analysis of surfactantenhanced remediation of denser-than-water nonaqueous phase liquid (DNAPL)contaminated soils. J Environ Qual. 2003;32(3):957-65.

74. Dubey R, Martini LG, Christie M. Duel-acting subcutaneous microemulsion formulation for improved migraine treatment with zolmitriptan and diclofenac: formulation and in vitro-in vivo characterization. AAPS J. 2014;16(2):214-20.

75. Aboofazeli R, Barlow D, Lawrence MJ. Particle size analysis of concentrated phospholipid microemulsions II. Photon correlation spectroscopy. AAPS PharmSci. 2000;2(3):E19. 
76. Fong WK, Hanley T, Boyd BJ. Stimuli responsive liquid crystals provide 'on-demand' drug delivery in vitro and in vivo. J Control Release. 2009;135(3):218-26.

77. Lee KW, Nguyen TH, Hanley T, Boyd BJ. Nanostructure of liquid crystalline matrix determines in vitro sustained release and in vivo oral absorption kinetics for hydrophilic model drugs. Int J Pharm. 2009;365(1-2):190-9.

78. Zeng Z, Zhou G, Wang X, Huang EZ, Zhan X, Liu J, et al. Preparation, characterization and relative bioavailability of oral elemene o/w microemulsion. Int $\mathrm{J}$ Nanomedicine. 2010;5:567-72.

79. Wall ME, Perez-Reyes M, Brine DR, Cook CE. Naltrexone disposition in man after subcutaneous administration. Drug Metab Dispos. 1984;12(6):677-82.

80. Xiao Q, Xiao C. Preparation and Characterization of Silica-Coated MagneticFluorescent Bifunctional Microspheres. Nanoscale Res Lett. 2009;4(9):1078-84.

81. Patel D, Sawant KK. Self micro-emulsifying drug delivery system: formulation development and biopharmaceutical evaluation of lipophilic drugs. Curr Drug Deliv. 2009;6(4):419-24.

82. Ornelas M, Azenha M, Pereira C, Silva AF. Naproxen-imprinted xerogels in the microand nanospherical formsby emulsion technique. J Chromatogr A. 2015;1422:43-52.

83. Araújo LM, Thomazine JA, Lopez RF. Development of microemulsions to topically deliver 5-aminolevulinic acid in photodynamic therapy. Eur $J$ Pharm Biopharm. 2010;75(1):48-55.

84. Andrióli, Tatiana Costa. Marcourakis, Tania (orient). Pacheco, Larissa Helena Lôbo Tôrres (orient). Exposição à fumaça do cigarro no início do período pós-natal : predisposição à dependência de drogas de abuso na adolescência. São Paulo, 2016. 116 p.

85. Niczinger NA, Hajdú M, Budai L, Dredán J, Antal I. [Importance of the HLB-value in drug technology]. Acta Pharm Hung. 2011;81(4):151-63.

86. Chorilli M, Prestes, Paula Souza, Rigon, Roberta Balansin,, Leonardi GR, Chiavacci, Leila Aparecida, \& Scarpa,, (2009). MV. Desenvolvimento de sistemas líquido-cristalinos empregando silicone fluido de co-polímero glicol e poliéter funcional siloxano. 2009.

87. Ali KA, Mukherjee B, Bandyopadhyay AK. Formulation development and in vitro evaluation of solidified self-microemulsion in the form of tablet containing atorvastatin calcium. Drug Dev Ind Pharm. 2013;39(11):1742-9.

88. Alkrad JA, Mrestani $\mathrm{Y}$, Neubert RH. Development and characterization of microemulsions containing hyaluronic acid. Eur J Pharm Sci. 2016. 
89. Krauel K, Girvan L, Hook S, Rades T. Characterisation of colloidal drug delivery systems from the naked eye to Cryo-FESEM. Micron. 2007;38(8):796-803.

90. Dubey R, Martini LG. Prolonged-release injectable microemulsions: Opportunities for pain treatment. Drug Development and Delivery. 2014;14(5):44-9.

91. Drummond CJ, Fong C. Surfactant self-assembly objects as novel drug delivery vehicles. Current Opinion in Colloid \& Interface Science. 1999;4(6):449-56.

92. Caboi F, Amico GS, Pitzalis P, Monduzzi M, Nylander T, Larsson K. Addition of hydrophilic and lipophilic compounds of biological relevance to the monoolein/water system. I. Phase behavior. Chem Phys Lipids. 2001;109(1):47-62.

93. Ganem-Quintanar A, Quintanar-Guerrero D, Buri P. Monoolein: a review of the pharmaceutical applications. Drug Dev Ind Pharm. 2000;26(8):809-20.

94. Lopes LB, Ferreira DA, de Paula D, Garcia MT, Thomazini JA, Fantini MC, et al. Reverse hexagonal phase nanodispersion of monoolein and oleic acid for topical delivery of peptides: in vitro and in vivo skin penetration of cyclosporin A. Pharm Res. 2006;23(6):133242.

95. Bouchemal K, Briançon S, Couenne F, Fessi H, Tayakout M. Stability studies on colloidal suspensions of polyurethane nanocapsules. J Nanosci Nanotechnol. 2006;6(910):3187-92.

96. Bouchemal K, Briançon S, Perrier E, Fessi H. Nano-emulsion formulation using spontaneous emulsification: solvent, oil and surfactant optimisation. Int $\mathrm{J}$ Pharm. 2004;280(1-2):241-51.

97. Ke WT, Lin SY, Ho HO, Sheu MT. Physical characterizations of microemulsion systems using tocopheryl polyethylene glycol 1000 succinate (TPGS) as a surfactant for the oral delivery of protein drugs. J Control Release. 2005;102(2):489-507.

98. Cichewicz A, Pacleb C, Connors A, Hass MA, Lopes LB. Cutaneous delivery of $\alpha-$ tocopherol and lipoic acid using microemulsions: influence of composition and charge. $\mathrm{J}$ Pharm Pharmacol. 2013;65(6):817-26.

99. Todosijević MN, Savić MM, Batinić BB, Marković BD, Gašperlin M, Ranđelović DV, et al. Biocompatible microemulsions of a model NSAID for skin delivery: A decisive role of surfactants in skin penetration/irritation profiles and pharmacokinetic performance. Int $\mathrm{J}$ Pharm. 2015;496(2):931-41.

100. Carvalho F, Sarmento, VH, Chiavacci, LA, Barbi,, MS G, MP. Development and in vitro evaluation of surfactant systems for, style="mso-bidi-font-style: crozJPSi, $2010 \mathrm{n}, 2367$ 2374. 
101. Diat O, Roux, D, Nallet, F. Effect of shear on a lyotropic lamellar phase, J Phys II 1993, 3(1427-1452).

102. de Silva, JP P, AS, Pansu, B, Davidson, P, Kasmi, B, Petermann, D, Asnacios, S, Meneau F, Imperor, M. Rheological behaviour of polyoxometalate-doped lyotropic lamellar phases. Eur Phys J E Soft Matter, 201134 (1): 1-9.

103. Amar-Yuli I, Wachtel E, Shalev DE, Aserin A, Garti N. Low viscosity reversed hexagonal mesophases induced by hydrophilic additives. J Phys Chem $B$. 2008;112(13):3971-82.

104. Feher A, Urban E, Eros I, Szabo-Revesz P, Csanyi E. Lyotropic liquid crystal preconcentrates for the treatment of periodontal disease. Int J Pharm. 2008;358(1-2):23-6. 105. Lara MG, Bentley MV, Collett JH. In vitro drug release mechanism and drug loading studies of cubic phase gels. Int J Pharm. 2005;293(1-2):241-50.

106. Siepmann J, Peppas NA. Higuchi equation: derivation, applications, use and misuse. Int J Pharm. 2011;418(1):6-12.

107. Siepmann J, Siepmann F. Mathematical modeling of drug release from lipid dosage forms. Int J Pharm. 2011;418(1):42-53.

108. Siepmann J, Siepmann F. Modeling of diffusion controlled drug delivery. J Control Release. 2012;161(2):351-62.

109. Yaghmur A, Glatter O. Characterization and potential applications of nanostructured aqueous dispersions. Adv Colloid Interface Sci. 2009;147-148:333-42.

110. Nilsson C, Edwards K, Eriksson J, Larsen SW, Østergaard J, Larsen C, et al. Characterization of oil-free and oil-loaded liquid-crystalline particles stabilized by negatively charged stabilizer citrem. Langmuir. 2012;28(32):11755-66.

111. Helledi LS, Schubert L. Release kinetics of acyclovir from a suspension of acyclovir incorporated in a cubic phase delivery system. Drug Dev Ind Pharm. 2001;27(10):1073-81. 112. Fong W-K, Hanley T, Boyd BJ. Stimuli responsive liquid crystals provide 'on-demand' drug delivery in vitro and in vivo. Journal of Controlled Release. 2009;135(3):218-26.

113. Patel N, Lalwani D, Gollmer S, Injeti E, Sari Y, Nesamony J. Development and evaluation of a calcium alginate based oral ceftriaxone sodium formulation. Prog Biomater. 2016;5:117-33.

114. Lopes CM, Lobo JMS, Costa PF, farmacêuticas de liberação modificada: polímeros hidrifílicos. Rev. Bras. Cienc. Farm. SP, v. 41, n. 2, p. 143-154,2005. J. 
115. Rossi NA, Reid,L.D. Affective states associated with morphine injections. Physiol.Pshicol.v4.p.269-276.1976.116. Cunningham CL, Gremel CM, Groblewski PA. Drug-induced conditioned place preference and aversion in mice. Nat Protoc. 2006;1(4):1662-70.

117. van der Kooy D, O'Shaughnessy M, Mucha RF, Kalant H. Motivational properties of ethanol in naive rats as studied by place conditioning. Pharmacol Biochem Behav. 1983;19(3):441-5.

118. Castro, LA, Baltieri, DA. The pharmacologic treatment of the alcohol dependence. Rev. Bras. Psiquiatr. São Paulo, v. 26, supl. 1, p. 43-46, May 2004.

119. Nascimento, Priscila Fernandes Carrara do. Efeitos da exposição ao etanol em camundongos adolescentes e adultos:comportamentos relacionados à recompensa, sensibilização comportamentale o papel dos sistemas dopaminérgico e glutamatérgico. 2011. Tese(Doutorado em Farmacologia) - Instituto de Ciências Biomédicas, Universidade de São Paulo, São Paulo, 2011. doi:10.11606/T.42.2011.tde-09022012-144606. Acesso em: 2017-07-3

120. Spragg, S.D.S. Morphine addiction in chimpanzees. Comp.Psychol.Monogr. v.15, p.132,1940.

121. Ray LA, Hutchison KE. Effects of naltrexone on alcohol sensitivity and genetic moderators of medication response: a double-blind placebo-controlled study. Arch Gen Psychiatry. 2007;64(9):1069-77.

122. Ray LA, Chin PF, Miotto K. Naltrexone for the treatment of alcoholism: clinical findings, mechanisms of action, and pharmacogenetics. CNS Neurol Disord Drug Targets. 2010;9(1):13-22.

123. Younger, J. \& Mackey, S. Fibromyalgia Symptoms Are Reduced by Low-Dose Naltrexone: A Pilot Study. Pain Medicine. Vol 10.N.4 (2009).

124. Simith, J.P. et al. Low-Dose Naltrexone Therapy Improves Active Crohn's Disease. Am J Gastroenterol 2007102:820-828.

125. Greenway, F.J. et al. Effect of naltrexone plus bupropion on weight loss in overweight and obese adults (COR-I): a multicentre, randomised, double-blind, placebo-controlled, phase 3 trial. www.thelancet.com Vol376 August 21, 2010. p.595-605.

126. Bruce A. C. Cree, Elena Kornyeyeva, and Douglas S. Goodin, Pilot trial of low-dose naltrexone and quality of life in multiple sclerosis. Ann Neurol.2010 Aug68(2):145-50. 